\title{
Trafficking of TNF via recycling endosomes in neutrophils
}

\author{
Nutan Srivastava*, Paige Lacy \\ From Canadian Society of Allergy and Clinical Immunology Annual Scientific Meeting 2014 \\ Ottawa, ON, Canada. 23-26 October 2014
}

\begin{abstract}
Background
Neutrophils are highly abundant innate immune cells that contribute to asphyxic episodes of acute asthma exacerbations, and secrete the proinflammatory cytokine tumour necrosis factor- $\alpha$ (TNF). Recycling endosomes (REs) are specialized secretory compartments that perform multiple functions including trafficking of cytokines to cell surfaces, although these are not characterized in neutrophils. Our objective is to identify trafficking components in neutrophils that may contribute to cytokine secretion.
\end{abstract}

\section{Methods}

The effect of bacterial lipopolysaccharide (LPS) stimulation on the trafficking of stored and newly synthesized TNF was determined by treatment of human peripheral blood neutrophils with or without cycloheximide. To visualize intracellular TNF, neutrophils were adhered to glass slides and treated with LPS for $1 \mathrm{~h}(10 \mathrm{ng} / \mathrm{ml})$. Colocalization of TNFa in neutrophils was performed with transferrin-Alexa 488 and anti-VAMP3 (markers for RE), anti-CD63 and anti-CD66b a membrane markers for the primary and secondary granules in neutrophils, respectively. We also determined Rab5 and Rab7 colocalization with TNF (markers for early and late endosomes, respectively). Imaging was carried out by Deltavision OMX super resolution microscopy.

\section{Results}

LPS induced 30-40\% TNF secretion from stored sources, with the remainder newly synthesized. We found that neutrophils possess REs as determined by transferrin uptake and VAMP3 labeling. TNF also colocalized with REs, primary and secondary granules as well as early and late endosomes, suggesting multiple sites of TNF storage and

\footnotetext{
Pulmonary Research Group, Department of Medicine, University of Alberta,
} Edmonton, Alberta, Canada and take full advantage of:

- Convenient online submission

- Thorough peer review

- No space constraints or color figure charges

- Immediate publication on acceptance

- Inclusion in PubMed, CAS, Scopus and Google Scholar

- Research which is freely available for redistribution 